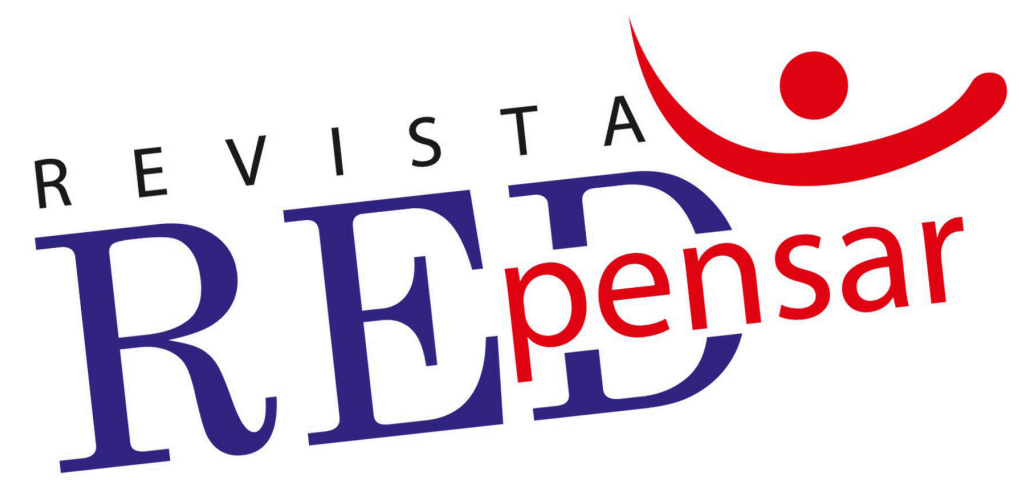

\title{
Hacia una Antropología del diálogo
}

Towards an Antbropology of dialogue

Ana Mariel Badilla Müllner

Revista REDpensar, volumen 7, número 2, Julio-Diciembre 2018 ISSNe: 2215-5384

REDpensando en la Investigación 2

Recibido: 20 de octubre de 2018 Aceptado: 29 de noviembre de 2018

DOI: 10.31906/redpensar.v7i2.168

\section{(ㄷ)(1)(2)}




\title{
Hacia una Antropología del diálogo
}

\author{
Towards an Anthropology of dialogue
}

Ana Mariel Badilla Müllner ${ }^{1}$

\section{Resumen}

El presente artículo parte de una breve pincelada sobre la realidad contemporánea para subrayar la necesidad de contraponer a la cultura globalizante contemporánea una cultura del diálogo, basada en un modelo antropológico relacional, abierto a la acogida plena de la diversidad. A partir de la certeza que "el otro", "el diferente", "el extranjero", no es una amenaza, sino una posibilidad de enriquecimiento. Concluye haciendo referencia al concepto de Verdad, el cual no es una realidad relativa, ni tampoco absolutista, sino relacional; nadie puede decir que la posee, más bien es Ella la que posee al ser humano en la medida que está vinculada con lo Absoluto.

Palabras Clave: DIÁLOGO, RECIPROCIDAD, VERDAD, RELACIÓN, AMOR, OTREDAD.

\section{Abstract}

This article begins with a brief brushstroke on contemporary reality to underscore the need to counterpose the globalizing culture contemporary to one culture of dialogue, based on a relational anthropological model, open to the full reception of diversity. From the certainty that "the other", "the different", "the foreigner" is not a threat, but a possibility of enrichment. It concludes by referring to the concept of Truth, which is not a relative reality, nor absolutist, but relational; no one can say who owns it, rather it is she who possesses the human being to the extent that is linked with the Absolute.

Keywords: DIALOGUE, RECIPROCITY, TRUTH, RELATIONSHIP, LOVE, OTHERNESS.

\section{Introducción}

Vivimos en una sociedad compleja y globalizada, caracterizada por el pluralismo y el universalismo. Mientras una parte del mundo tiende a reivindicar identidades nacionales o locales, como subraya el emblemático caso del Brexit o la lucha por la independencia del Estado Catalán, contemporáneamente hay una fuerte exigencia de propuestas integrales, de garantizar derechos universales a todos los seres humanos del planeta.

1. MEd. en Tecnología Educativa con énfasis en diseño de material instruccional, Universidad Estatal a Distancia [UNED]. Docente del curso de Métodos de Investigación, Colegio Universitario de Cartago [CUC], y del curso Diálogo como cultura, en Universidad Católica de Venezuela, Maracaibo. Estudiante de Doctorado, Universidad La Salle. Correo electrónico: mariel.badilla@gmail.com 
Se multiplican las cumbres, las conferencias mundiales, las organizaciones internacionales en aras de encontrar respuestas globales para las distintas problemáticas contemporáneas, pero al mismo tiempo somos testigos de una especie de globalización del terror [cursivas propias de la autora], que se expresa a través de la consolidación de regímenes totalitaristas y del sistemático atentado contra los derechos humanos ante la impotente e ineficaz condena de la comunidad internacional; el poderío de los cárteles internacionales de narcotráfico, de conflictos religiosos sin fundamento, se verifican en poblaciones donde los distintos credos siempre vivieron en paz, y repentinamente se convierten en protagonistas de terribles masacres. Especialmente preocupante es la creciente ola de actos terroristas y la persecución religiosa que se ha desatado en todo el planeta.

A esta realidad se suma, como una de sus más tristes consecuencias, un fenómeno migratorio de enormes proporciones, al que están vinculadas otras y muchas problemáticas, como la xenofobia, la construcción de muros y políticas de exclusión, la trata de personas, la persecución y el odio hacia quien es diferente por nacionalidad, religión, sexo o raza. El discurso de la diversidad, de hecho, está vinculado a temas álgidos y urgentes.

La diversidad, tal como explica Schiavo (2012), "hace parte de la naturaleza misma de las cosas" (p.89). Las variaciones genéticas, biológicas, culturales, climáticas, históricas, sociales, religiosas, entre otros, generan y provocan variaciones en las especies y en los grupos sociales. Tales variaciones se transforman en características específicas que dan vida, entre otras cosas, a culturas diferentes, necesarias para la definición de las identidades sociales. En el campo social y cultural las diferencias pueden ser de normas, usos, costumbres, cosmovisión, lenguaje y sistemas simbólicos. Schiavo (2012) afirma que en un sistema globalizante como el que caracteriza la cultura contemporánea,

[...] las diferencias pueden transformarse en factores potenciales de conflicto. Porque la diversidad marca fronteras y límites entre un dentro y un afuera, un semejante y un distinto, un próximo y un lejano, un familiar y un extraño (extranjero), un conocido y un desconocido, un amigo y un potencial adversario. (p. 89)

¿Qué está detrás de este rechazo epidérmico del ser humano a todo lo que puede resultar diferente? Las explicaciones y perspectivas desde las cuales se puede analizar este fenómeno son numerosas y no resulta posible hacer un análisis exhaustivo en este artículo; sin embargo, resulta pertinente describir sintéticamente el humus en el que se desarrollan estas manifestaciones de intolerancia y temor hacia la diversidad cultural.

\section{La cultura globalizante y la desarticulación de lo diverso}

El fenómeno de la globalización que originalmente estaba vinculado a un proceso económico, de matriz neoliberal, mediante el cual se pretendía crear un único mercado libre de cualquier tipo de barrera, se ha convertido en un proceso complejo que ha invadido numerosas esferas. De hecho, dadas las características de este fenómeno se puede afirmar que la globalización es en sí misma una cultura, es una nueva forma de entender la vida de las personas y sus relaciones. Se trata de un fenómeno en el cual podemos encontrar numerosas posibilidades de desarrollo humano, pero al mismo tiempo está vinculado a un grave peligro: la globalización cultural.

La misma está basada en la lógica del mercado, que a su vez está vinculada a una cultura individualista, donde la única cosa que cuenta es el éxito a cualquier costo. Se trata, afirma Araujo (2007) de una cultura del conflicto, que invita a los ciudadanos al contraste y a una competencia desenfrenada y tiende a hacer desaparecer las diferencias y las culturas locales, pues se rige por criterios homologantes que tratan de imponer un único pseudo-pensamiento, una única pseudo-moral y una pseudo-visión de los acontecimientos, abatiendo, como si fuese una aplanadora, la riqueza y la variedad de las diversas culturas. 
Se parte de una visión cultural etnocéntrica, que ignora o menosprecia cualquier realidad cultural diferente, en la convicción de que todo lo que no coincide con los valores de la cultura dominante, ha de ser erradicado. El etnocentrismo es antiguo como la raza humana y lamentablemente en la historia abundan los ejemplos de estos procesos de desarticulación cultural de pueblos y de naciones; sólo que, precisamente por las características globales de nuestro tiempo, se ha llegado a una degeneración de tal envergadura que algunos autores como Buber (1983) y Heschel (1987) citado en Pérez (2007, p.55) han designado este momento histórico con el dramático título de "eclipse de la humanidad".

Paradójicamente, el mismo fenómeno de la globalización, ha aumentado exponencialmente las posibilidades de encuentro entre personas y pueblos diferentes, y ha contribuido a poner en tela de juicio la perspectiva etnocéntrica, abriendo paso a una profunda reflexión sobre la realidad del Otro como elemento esencial para el desarrollo humano.

En este esfuerzo se destacan algunos pensadores de matriz judeo-cristiana como Rosenzweig, Martin Buber, Lévinas, Emmanuel Mounier, quienes ya en el siglo pasado empezaron a desarrollar un nuevo paradigma antropológico de carácter personalista, que supera la visión individualista y etnocéntrica, y se fundamenta en el diálogo y el reconocimiento de la alteridad.

\section{E1 "Alter" como otro yo}

Según el filósofo Emmanuel Lévinas, quien fue un sobreviviente del holocausto, el sujeto humano debe ser abordado de otro modo, diferente del saber objetivante, es decir desde su alteridad o su otredad, en la cual se manifiesta y revela su subjetividad independiente y libre.

Explica Rabinovich (2000), citada por Lévinas (2000) que

La objetivación del otro es la suprema tiranía, donde las diferencias son eliminadas y todo es reconducido al 'Yo' [haciendo referencia directa al holocausto, el autor recuerda que] "la víctima era uniformada, rasurada, su piel era grabada con un número, todo era cuestión de borrar lo particular y lo expresivo de su rostro". (p.30)

Es a partir de aquí que Lévinas (2000) citado en Schiavo (2012) plantea su propuesta

Es necesario repensar la ética desde el otro, una ética que respete y valorice la otredad, su subjetividad, que proponga la responsabilidad por el otro como principio supremo capaz de desestructurar el imperialismo del "yo", y de garantizar la libertad desde la heteronomía y no desde la autonomía, es decir desde la relación con el "otro". (p.101)

Cicchese (1999) citado en Luna (2014) coincide con esta perspectiva cuando afirma que la relación es el fundamento antropológico y ontológico de la naturaleza humana. No existe un solo acto de la existencia en el cual se pueda estar totalmente solo. "El hombre no debe estar cerrado solo en los límites estrechos de una árida subjetividad, sino abierto a una dimensión relacional" (p.3). En esta misma línea Buber (1923) citado en Luna (2014) dirá: "cuando digo yo, digo tú; cuando digo tú, digo yo" (p.3).

Para Buber, de hecho, el objeto central de la antropología y de la sociología no es ni lo individual ni lo colectivo, sino el-hombre-con-el-hombre.

El Yo-Tú existe solamente en el mundo del hombre, y todavía más, el Yo existe solamente en la relación con el Tú. La ciencia filosófica del hombre, que incluye la antropología y la sociología, debe, por lo tanto, tomar como punto de partida, como objeto de investigación, el-hombre-con-elhombre. (Buber, 1983, pp. 124-125). 
Siguiendo esta misma línea de pensamiento Emmanuel Mounier, desafiando el principio cartesiano que había puesto en el pensamiento la esencia del ser, cogito ergo sum (pienso, luego soy), propone que es el amor el elemento fundamental de la naturaleza humana: Amo ergo sum (amo, luego soy) escribe Mounier (Fons, 2006, p.16). El reconocimiento del Otro, por lo tanto, resulta esencial en el camino de personalización del ser humano.

A partir de los aportes de estos pensadores podríamos vislumbrar algunos elementos fundamentales de un nuevo paradigma antropológico, que, superando la concepción individualista de la modernidad, se fundamenta en el cambio de la relación y sostiene que es mediante el encuentro con el Otro que el humano se desarrolla como persona.

Hablamos de una perspectiva que no se limita al reconocimiento del otro o a la aceptación y respeto de la diversidad, sino que reconoce en ella una posibilidad ulterior de enriquecimiento. El Otro entonces, no sólo no representa una amenaza, sino que es necesario para la realización del ser humano, es más, es el Otro quien se vuelve constitutivo del ser, en la medida que se le considera, como señala la tesis de Voce (2014): "Otro Yo”. El vínculo que hace posible este tipo de relación como señala Mounier es el amor, que es el fundamento de la reciprocidad.

\section{Reciprocidad y paradigma relacional}

En las relaciones humanas hay siempre una doble vía, afirma Pozzi (2011) citado en Luna (2014, p.4) "la otra persona "me interpela con su sola presencia, me llama a la responsabilidad" igualmente mi presencia interpela e invita al otro a su responsabilidad en relación conmigo, naciendo de esta manera la reciprocidad".

El Otro, como señala Cicchesse, es mi correctivo antropológico, porque es él quien marca mis confines y me ayuda a definirme como persona.

La práctica de la reciprocidad lleva al encuentro con la(s) otra (s) persona (s), "es algo más que la suma de los o las participantes. Generar encuentro implica la "aceptación incondicional positiva de la otra persona”, es decir aceptarla tal cual es, darle un recibimiento caluroso y empático". (Luna, 2014, p.5)

Sin embargo, si queremos hablar de auténtica reciprocidad es necesario un paso ulterior. La relación auténtica con el Otro va más allá de la aceptación incondicional y positiva, implica abrirse, donarse al Otro, perderse en el Otro.

Se trata de un concepto que podría parecer una paradoja, en una sociedad como la nuestra en la que más bien las personas tienden a atrincherarse en su espacio para protegerse su identidad y garantizar sus derechos individuales, cerrando así las puertas de los Estados y de los corazones.

Sin embargo, este "perderse en el otro" es precisamente el núcleo central de la desafiante propuesta que conlleva una antropología relacional. Desde esta perspectiva se invita al ser humano a salir de su subjetividad, para donarse totalmente, descubriendo en este paso la posibilidad de auto-trascenderse.

Para explicar esta experiencia, Cicchese (2011), acuña el concepto de la "apropiación expropiante", que básicamente invita a la persona a salir de sí misma para acoger al Otro, en un ejercicio de "pérdida" de la propia perspectiva, para enriquecerla con la perspectiva del Otro. 
La esencia de esta dinámica relacional que lleva a la reciprocidad como se citó anteriormente es el amor, que ofrece y genera amor cuando es auténtica. Muy bien lo explica Karl Marx al decir: "cuando tu amor no produce amor recíproco y el manifestar tu vida de hombre que ama, no hace de ti un hombre amado, tu amor es impotente, es una desgracia" (Voce, 2014, p.274).

En otras palabras, podríamos decir que la prueba de la autenticidad de una relación basada en el amor está precisamente en su capacidad de suscitar en el Otro una respuesta de amor.

\section{Consecuencias fenomenológicas}

A partir de lo dicho anteriormente resulta importante aclarar qué se entiende por amor y cuáles son sus consecuencias fenomenológicas en las relaciones humanas. Para explicar sus características esenciales haremos referencias a las categorías utilizadas por Lubich (1982), cuando describe lo que ella denomina "arte de amar".

El amor verdadero, afirma Lubich (1982), es universal, no parcial, no excluyente según los gustos y categorías de cualquier tipo: raza, color de piel, nacionalidad, religión, etc. Todos deben estar incluidos en el circuito del amor.

El cual nos libera de todas las esclavitudes. Porque somos esclavos de las divisiones entre pobres y ricos, entre generaciones, entre razas, entre nacionalidades, hasta entre Estado y Estado nos criticamos, existen obstáculos, barreras. ¡No! La primera idea es liberarnos de toda esclavitud y descubrir en todas las personas posibles candidatos a la fraternidad. (Lubich, 1982, p.2).

Es capaz -explica siempre Lubich (1982)- de tomar la iniciativa, es decir, de superar las barreras que comportan la diversidad cultural; además, sabe ir más allá del individualismo que caracteriza la sociedad contemporánea para ir en busca del Otro, no se deja condicionar por los prejuicios, ni por el temor ante la diversidad.

La tercera consecuencia del amor, según Lubich (1982), es la capacidad de ponerse en el lugar del otro. Traduciendo en acciones concretas la máxima que reza: 'haz a los demás lo que te gustaría que te hicieran a ti y no hagas lo que no te gustaría que te hicieran' (Mt 7,12). O como dice Mahatma Gandhi: "tú y yo somos una sola cosa, no puedo hacerte daño sin herirme".

La actuación de este principio, implica una especie de "deconstrucción”, como la denomina Mazzola (2010), del propio esquema mental y las propias categorías culturales, "suspendiéndolas" por un momento, para hacer el esfuerzo de entender y sentir las cosas desde la perspectiva del Otro. Se trata del desafiante proceso de perder, de no-ser, para estar en grado de acoger plenamente al otro, en una vivencia radicalmente empática, que en la práctica suele terminar siendo una fuente de enriquecimiento y ampliación de la propia perspectiva.

Finalmente Lubich (1982) explica que una cuarta consecuencia del amor es la anulación de la categoría del enemigo, que lleva a excluir la contraposición, el conflicto, el uso de la fuerza, la imposición del poder, la explotación y la opresión, y abre espacios al perdón y a la reconciliación.

\section{Cultura del diálogo y ética del encuentro}

El fundamento cultural del paradigma antropológico antes descrito está estrechamente vinculado a una ética basada en el diálogo y el encuentro. La cultura del diálogo es, de hecho, el bumus natural donde puede desarrollarse el ser-en-relación [cursiva de la autora], es el medio a través del cual ponemos en práctica el arte de amar del que hemos estado hablando y la ética del encuentro, viene a ser la aplicación de dicha cultura.

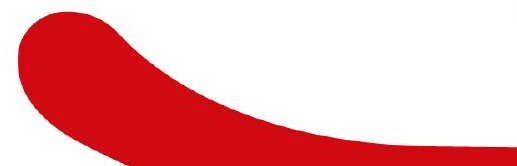


La raíz etimológica de la palabra diálogo ofrece elementos importantes para comprender este proceso humano. El término proviene de las palabras dia (a través de) y logos, un concepto que contiene varios significados que los idiomas modernos han separado: puede significar entre otras cosas palabra, razonamiento y sabiduría. El término hace pensar en un camino conjunto hacia una meta utilizando palabras y razonamientos.

Según Bohm (2001), el diálogo es un proceso, que parte de los puntos de vista individuales, y va fluyendo libre, mediante la conformación de un sentimiento de comunidad donde "puede aparecer un nuevo tipo de mente, basada en el desarrollo de un significado común, que va transformándose de continuo gracias al proceso de diálogo" (p. 13).

Se trata de un proceso desafiante, porque implica superar las opiniones o suposiciones individuales y abrirse a la diversidad del Otro, suspendiendo, como se explicaba anteriormente, nuestros juicios y creencias para acoger la riqueza que puede aportar el contacto con los otros participantes en el diálogo.

Williams (1999) citado en Bolívar (2007) afirma que

El diálogo es la manera de asumir las energías de nuestras diferencias y canalizarlas hacia algo que nunca ha sido creado antes. Remplaza la lógica del pensar -por- tu cuenta con la del pensar-con-los-demás que permite descubrir valores compartidos entre las partes, y hace que podamos coordinar y alinear con ellos nuestras acciones y nuestros pensamientos. Por supuesto, para llegar a eso, es indispensable renunciar a la idea que nuestra opinión sea la más correcta y que sea la que quede al final del diálogo. (p.59)

El diálogo, por lo tanto, no es sencillamente una negociación, una estrategia para llegar a un acuerdo, una manera lo más educada posible de conversar y aplanar divergencias. Presupone la existencia de una diferencia, el reconocimiento de éstas como un valor y el querer aprovecharlas para la creación de algo nuevo e inesperado. Las opiniones personales son importantes y, si son amenazadas, hay que defenderlas, pero tienen valor solamente en la medida en que cada quien haga el esfuerzo de poner a disposición del Otro la propia y trate de entender lo más posible las de los demás.

Ahora bien, lo que conceptualmente acabamos de describir, se traduce en actitudes precisas o en lo que podríamos denominar, como se dijo anteriormente, una "ética del encuentro". El concepto lo desarrolla Schiavo (2012) en el tema de la "Otredad" y la tendencia a vincularla con el imaginario del diablo. Desde su perspectiva Schiavo propone 5 actitudes, que se citan en forma sintética a continuación, necesarias para superar la visión errática del Otro como una amenaza:

- Apertura al extraño/extranjero: en un mundo que busca seguridad, tranquilidad, paz, refugio, no se debe tener miedo del extraño, sea él el forastero, el migrante, el homosexual, o simplemente un comportamiento diferente. Mirar de frente al extraño es dejarse incomodar por su extrañeza, aceptar salir de lo "normal", ponerse en juego y enfrentar al desconocido, al nuevo y la incertidumbre. Esta actitud lleva a la transformación perenne del ser, a la conciencia de que nada es definitivo, pues la vida es camino. En ese sentido, las puertas nunca se cerrarán frente a nadie.

- Disponibilidad al desarraigo: el extraño nos saca fuera, el encuentro con el otro quiere decir desarraigo, estar siempre en viaje. Es un proceso de auto-extrañeza, que provoca inquietud, cambio continuo, búsqueda, deseo, angustia, fatiga, perseverancia. Porque la vida es relación, encuentro, camino: sin el otro no hay vida: quedarse cerrados en sí mismos o en su propio grupo equivale a la muerte: la muerte del prójimo y ¡mi propia muerte! Porque el uno, sin el otro, no es nada. Lo que define la identidad es justamente la relación: somos las relaciones que vivimos.

- Buscar el "más allá": las palabras, también las idénticas, no tienen el mismo significado. Acoger la diferencia es renunciar a poseer, a apoderarse, a dominar; es admitir que el misterio 
del otro está siempre más allá de lo que percibimos, y que siempre se escapa de nuestras manos. Al final, la verdad es siempre el resultado de un proceso inacabado y colectivo, producto de las relaciones: nunca un dogma definido e impuesto una vez por todas.

- Actitud de silencio y escucha: la presencia del Otro impone el silencio, la escucha, para que él pueda manifestarse. Escucha de lo que el Otro trae, es, vive: es silenciar nuestro yo para dar espacio al Otro. El encuentro genera así la compasión, que no es pena, sino sintonía, vibración a la misma onda. De la compasión nace la simpatía (de sun-pathos, sentir juntos). El Otro es traído para dentro, es parte del Yo y el Yo es parte de él. La otredad no es más problema, barrera, muro de separación, porque la proximidad posibilitó el encuentro. Si la indiferencia proporciona una paz ilusoria, el encuentro impone la responsabilidad y la solidaridad, porque el Otro ya es parte de mí.

- Apertura a la trascendencia: la transcendencia pide una mirada diferente, capaz de ir más allá de las apariencias y, sobre todo, de hablar otra lengua: la del misterio que cada persona trae consigo.

En el camino de la transcendencia -dice- tres son las puertas por las cuales hay que pasar: la puerta que lleva a salir de sí mismo rumbo al Otro; la puerta que permite la entrada en el Otro, y lleva a la aceptación de su otredad; y, finalmente, la puerta que abre hacía el Otro que siempre está más allá, como el eterno Presente, que es el sentido de todo. (Schiavo, 2012, p. 132)

En estos $\mathbf{5}$ puntos encontramos elementos esenciales para pasar de una reflexión teórica sobre el ser humano, sobre la alteridad y sobre el concepto de diálogo, al esbozo de un estilo de vida dialógico aplicable en la cotidianidad de todas las personas. Sin embargo, resulta importante destacar que el último punto (apertura a la trascendencia) es el que nos abre a la novedad implícita en esta tesis, porque en el atrevimiento de referirse al "Otro que siempre está más allá" [cursiva de la autora], rompe abiertamente con la perspectiva atea que impregna el pensamiento contemporáneo para devolver el espacio a lo espiritual y a lo trascendente.

Porque sólo la conciencia de que en el encuentro con el Otro nos encontramos con Dios, haciendo factible un estilo de relación como el que hemos planteado.

Antes de concluir esta reflexión es importante retomar un último punto. ¿Cómo se vinculan todos estos elementos con el concepto de verdad?

\section{La verdad relacional}

No se puede hablar de diálogo y del enriquecimiento que suscita la diversidad sin afrontar el concepto de verdad.

El diálogo auténtico es una profundización de la verdad. De hecho, para los griegos el diálogo era el método para llegar a la verdad. Esto quiere decir que la verdad siempre necesita ser completada, no se trata de relatividad de la verdad, sino de "relacionalidad de la verdad", explica Baccarini (s.f) citado por Morán (2016). Por lo tanto, la verdad no es ni relativa ni inexistente, es relacional.

Verdad relacional quiere decir que cada uno participa de la verdad, que es una. En la relación cada uno descubre aspectos nuevos de la verdad como si fueran suyos. La Relatividad de la verdad, en cambio quiere decir que cada uno tiene su verdad. (Morán, 2016, p. 5)

Panikkar, gran maestro del diálogo que vivió por mucho tiempo en India, citado por Morán (2006), decía: "desde una ventana se puede ver todo el paisaje, pero no totalmente", es decir, todas las perspectivas aportan a la verdad, se complementan, el acercamiento a la verdad pasa entonces por la relación.

Afirma el teólogo italiano Coda (1988), retomando el concepto mencionado anteriormente del perder y vinculándolo al tema de la verdad,

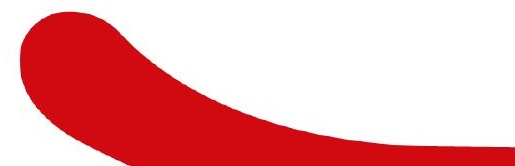


Si en el diálogo con cada ser humano, puedo realmente ponerme en juego a mí mismo, perdiendo aquel pedacito de Verdad que había entendido, entonces sí la Verdad podrá poseerme con más profundidad y podrá misteriosamente "arrastrar" consigo también a mi interlocutor. Si en cambio permanezco "celoso" de mi verdad, nunca llegaré a la Verdad; si la pierdo, en cambio me perderé a mí mismo, perderé mi media verdad y podré encontrar la Verdad. (p. 113)

La verdad no es relativa, o inexistente, tampoco es cierto que cada quien tiene su verdad y basta. Esta tesis podría resultar sumamente peligrosa, pues empuja al ser humano al relativismo moral, a la carencia de puntos de referencia que termina diluyendo la identidad personal y cultural del individuo.

La Verdad existe, pero, como se señaló anteriormente, nadie puede decir que la posee, porque es más bien Ella quien nos posee. La Verdad no es una cosa, o una idea, es una Persona y se identifica con ese Otro, Eterno Presente del que hablamos anteriormente. Para el cristiano se refiere a Aquel que dijo de sí mismo: "Yo soy el camino, la Verdad y la vida" (Jn 14,6).

\section{Referencias Bibliográficas}

Araujo, V. (2007). Reflexiones sobre la Globalización. Seminario de formación. Roma, Italia.

Bohm, D. (2001). Unfolding meaning. En L. Nichol, Sobre el diálogo. Barcelona, España: Kairos.

Bubber, M. (1923). Yo y tú. Barcelona, España: Editorial Herder.

Buber, M. (1983). Il problema dell'uomo. Turín, Italia: Elle Di Ci.

Cicchese, G. (1999). I percorsi dell'altro. Antropologia e storia. Roma, Italia: Città Nuova.

Cichesse, G. (2011). Antropología del diálogo. Buenos Aires, Argentina: Ciudad Nueva.

Coda, P. (1988). Verità - Dialogo - Trinitá. Dialogo fra le culture. Chiesa e umanesimo planetario. Roma, Italia: Città Nuova.

Heschel, A. (1987). El judaísmo como problema personal, Democracia y otros ensayos, Buenos Aires, Seminario Rabínico Latinoamericano. p.125.

Lévinas, E. (2000). La buella del otro. Ciudad de México, México: Taurus.

Lubich, C. (1982). La unidad. Italia: Centro Chiara Lubich. Recuperado de www.centrochiaralubich. org/pt/pdf/espanol/522-chi...es/file.html

Luna, A. (14-17 de octubre de 2014). Perspectivas de los Político desde la Fraternidad. En VI Seminario Internacional sobre estudios de fraternidad. Hacia una antropología relacional. Seminario llevado a cabo en Bogotá, Colombia: Universidad Santo Tomás.

Mazzola, C. (2010). Oltre il pregiudizio, all'accoglienza all'integrazione. Percorso Formativo "Cambia... Menti" La Scuola italiana e le sfide della globalizzazione. Roma, Italia: Associazione Azione per un Mondo Unito ONLUS.

Morán, J. (2016). Alcuni aspetti antropologici e culturali del dialogo. Universidad de Mumbai, India. Recuperado de https://bit.ly/35mjEwk 
Pérez, V. (2007). La religión judía desde Buber y Heschel ante la postmodernidad. Historia y Grafía, (28), 41-68.

Pozzi, R. (2011). Ripensare l'uomo e l'ethos: il contributo della riflessione filosofica di Edith Stein. En A. Luna (expositor), Perspectivas de lo político desde la Fraternidad (conferencia). Bogotá, Colombia.

Schiavo, L. (2012). La invención del diablo. Cuando el otro es un problema. San José, Costa Rica: Lara Segura y Asociados.

Voce, M. (2014). La visione dell'uomo in Chiara Lubich. Nuova Umanità, 3(213), 269-281.

Williams, I. (1999). Dialogue: The Art of Thinking Together. Nueva York, Estados Unidos: Doubleday. 